\title{
A Study on the Making Indoor Topology Map for the Autonomous Driving of Wearable Robots
}

\author{
Se-Yeob Kim, Researcher, Computer Engineering, Sunmoon Univ., ASAN, KS002, Republic of Korea, \\ kimseysob@gmail.com \\ *Yoon-Young Park, Professor, Computer Engineering, Sunmoon Univ., ASAN, KS002, Republic of Korea, \\ yypark@sunmoon.ac.kr \\ Kyung-Oh Lee, Professor, Computer Engineering, Sunmoon Univ., ASAN, KS002, Republic of Korea, \\ leeko@sunmoon.ac.kr \\ Ji-Bum Jung, Master Student, Computer Engineering, Sunmoon Univ., ASAN, KS002, Republic of Korea, \\ 2377325@gmail.com \\ Joong-Eup Kye, Professor, Computer Engineering, Sunmoon Univ., ASAN, KSO02, Republic of Korea, \\ jekeh66@naver.com \\ *Corresponding Author
}

\begin{abstract}
When walking with a wearable robot, we may not react properly to the surrounding environment. Therefore, we need to help the wearable robot to walk correctly by providing information of the surrounding environment in the indoor space. SLAM creates a map while the robot moves in real time, so you can use the same map as the actual structure, but it takes a lot of time to create the map. We produce a topology map using the floor plan image. Since the floor plan is produced when a building is made, it is very similar to the actual structure of the building and it is very efficient in terms of time because it does not need to create a map in real-time. In addition, since we generate our topology map using the sensor nodes installed in the building, it matches the coordinates on the floor plan one to one so that the path can be set more accurately and we can find doors, stairs, and obstacles more easily found through object detection. In this study, create a topology map based on a floor plan so that a wearable robot can walk more stably and autonomously, and we can provide the information of object detection by learning a dataset of rooms, doors, and stairs. By synthesizing this information, we transmit it to the wearable robot and provide a path to progress. Conduct an experiment to detect obstacles in the path and transmit the information to wearable robots to prevent accidents.
\end{abstract}

Keywords: Indoor Navigation System, Simulator, Feature Detection, Wearable Robot, Topology map $\begin{array}{lll}\text { Received: 08.12.2020 } & \text { Accepted: 11.01.2021 } & \text { Published: 06.02.2021 }\end{array}$

\section{INTRODUCTION}

In recent years, wearable robots, which have been at the center of rehabilitation and medical care, are increasingly being introduced into industrial fields such as logistics, manufacturing, construction, and services in order to improve the working environment and the problem of aging and a decrease in the working population. In addition to the exoskeleton type wearable robots, which are mainly used in the field of rehabilitation and defense, research and development for soft wearable robots are also underway to satisfy conditions such as light, flexible, easy to wear, low noise, and low price[1].

In recent years improve the prediction capabilities of lower-limb locomotor intent recognition systems; ideally, providing a framework for autonomous wearable robots which can assist wearers with a diverse range of activities encountered in the real-world[2].

As such, research on autonomous driving of wearable robots is gradually progressing. However, in the case of paraplegia or general paralysis, the lower body cannot be moved by itself. That's why you have to wear a wearable robot and walk. Especially if you walk indoors, not outdoors, wearing wearable robots, it can be difficult to change directions, and hurt by hitting obstacles. Because the building has become more complex recently[3].

A lot of research has been conducted to create a topology map using a floor plan. In the past, when creating a topology map, a route is created using hop count within the communication range of each sensor, but in this study, designate a sensor type, and establish a path by connecting to a sensor node of another type within the communication range of each sensor. In order for the wearable robot to freely travel more safely and flexibly, a Topology Map is produced based on an indoor map, that is, a building floor plan, and the wearable robot is provided with a path of the wearer. If there is an obstacle in the path, 
research is conducted to detect the obstacle and transmit the information to the wearable robot.

\section{Related Works}

Recently, research on an indoor navigation system that finds a way inside a building is being actively conducted.

\subsection{Simulation Framework for Evaluation of Indoor Navigation Systems}

Introduce the first simulation framework that provides cost-effective means to evaluate indoor navigation systems for different user groups, various positioning techniques, and navigation instructions algorithms [4].

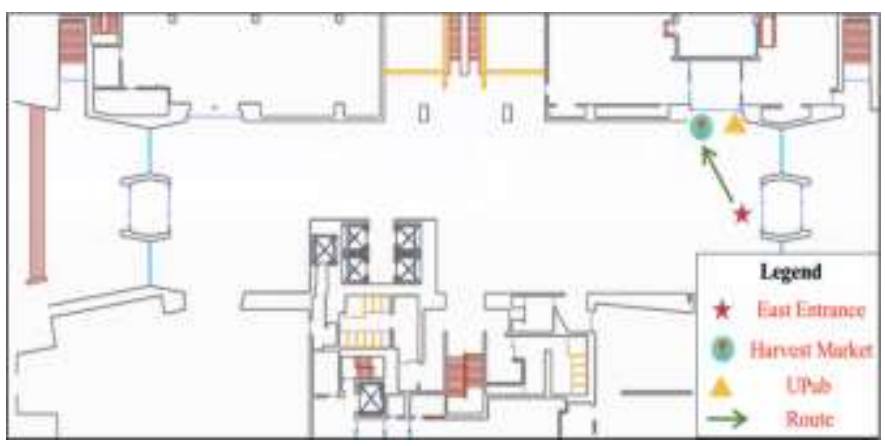

Figure 1. Center Virtual Environment

Fig. 1 is a virtual environment for the central entrance floor of the UMASS campus created in Unity. In this study, it was proposed to develop and test VUM (Virtual User Model) that simulates users. VUM simulates the user's space Navigation Behavior in a virtual environment, and the virtual user understands the navigation instructions and acts by recognizing the surrounding environment.

However, in the case of this study, a study was conducted on the route guidance to the landmark inside the building, and only the route leading to the entrance of the landmark was exhibited. It is regrettable that it does not reflect the actual environment inside the building. In this study, it is an advantage that more precise guidance can be provided because the path is guided by reflecting the location of each room on the floor plan and the actual internal environment.

\subsection{IndoorWaze: A Crowdsourcing-Based Context-Aware Indoor Navigation System}

Research has been conducted to propose IndoorWaze, a new crowdsourcing-based situation-aware indoor navigation system that can accurately recognize the situation using the labeled points of interest(POI) and automatically produce a floor plan[5].

IndoorWaze combines the Wi-Fi fingerprint of indoor pedestrians and the label provided by POI to produce a high-reliability floor plan. IndoorWaze all stores are properly labeled and placed. You can also create a high-confidence labeled floor plan with all routes and intersections displayed correctly and an average estimate error of less than $12 \%$ for the store.

However, in order to obtain a route, there is a disadvantage that people must track and collect the paths they walk around. That is, if a new place is created or the surrounding environment changes, there is a problem in that it is necessary to additionally track and collect the walking paths of people. In this study has the advantage of not being greatly interfered by the surrounding environment because it guides the path by synthesizing the information of sensor nodes without the task of tracking the path.

\subsection{Submap-Based Indoor Navigation System for the Fetch Robot}

In order to cope with large-scale environments or multi-story buildings, research has been conducted using a submap mapping framework that combines a 2D laser scan and a 3D point cloud of RGBD sensors[6]. RGBD Multiple image datasets of the pose are created through the RGBD sensor. In addition, accuracy is improved because errors are limited according to the size of the map due to the submap framework. 


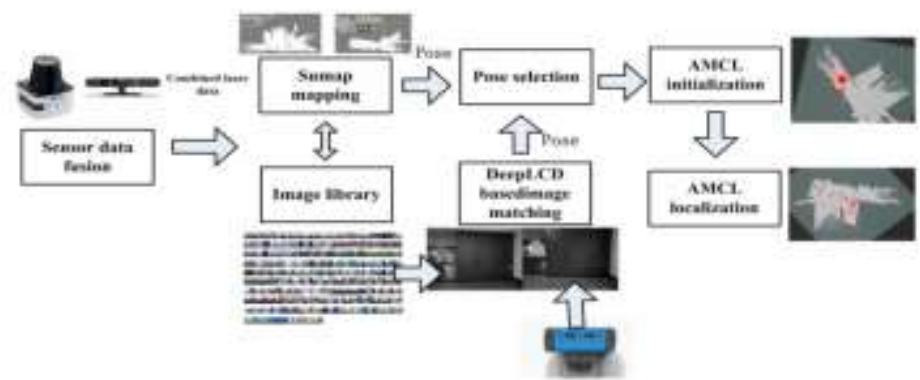

Figure 2. Whole Solution framework

The online image of the RGBD sensor is used to convert the submap for online localization. It also matches database images using DeepLCD, a deep learning-based library for loop closure. Fig. 2 is a flow chart of the proposed overall solution framework.

Since the 2D laser scan and RGBD sensor are used, actual data can be obtained, but there is a problem in synchronizing because the coordinate system, type stamp, and frequency are different. And it takes a lot of time to complete the indoor map because it is necessary to obtain information about the map while moving by introducing a robot.

As described above, until recently, numerous studies have been conducted on indoor navigation systems. However, research on indoor navigation systems for people with walking restrictions is still insufficient. Therefore, this study aims to implement an indoor navigation system that can guide a safe route by matching the actual surrounding environment and the indoor map for people who are restricted in walking.

\section{Implementation}

In the indoor space, there are structures that do not exist in the outdoor space, such as doors, corridors, and stairs, so the indoor space is more restricted in movement than the outdoor space. These restrictions may also exist in outdoor spaces, but more types of restrictions can be dynamically changed since indoor spaces can be reorganized relatively easily compared to outdoors[7].

Among various types of indoor spatial information services, a service provided using indoor positioning information based on a specific location model and coordinate system in an indoor space is called an indoor location-based service. This service has become the core of various services targeting indoor spaces[8].



Figure 3. Flowchart of Topology Map Creation

Fig. 3 shows the overall flow chart of creating a topology map. Until recently, an indoor map was generally create using SLAM technology, and based on this map, the final topology map was created by overlaying it with a sensor network. In the method of creating a map using SLAM, since the robot moves in real time and creates a map, the same map as the actual structure can be used, but it takes a lot of time to create the map[9].

The advantage of this work is that the map is created in real time, so it is the same as the real building environment, and changes can be applied in a short time. The disadvantage is that it takes a lot of time to generate the map. Also, making indoor maps using SLAM is expensive. In order to obtain map information, a robot that moves autonomously and a LiDAR sensor are required. However, in order to create a map, not only one robot is used, but several robots must be introduced. In order to put in these equipments, it is 
financially burdensome.

In this study, the topology map is generated using the image of the building floor plan. This method creates a topology map by matching the floor plan image $1: 1$ with the coordinates of the actually attached sensors. Then, it detects rooms, doors, and corridors on the floor plan using object detection. Since the floor plan is a map created when the building was actually built, it is very similar to the actual building structure, and it is very fast in terms of time because there is no need to generate the map in real time. And unlike the method of creating an indoor map using SLAM, the advantage is that there is little financial burden because it only needs a floor plan image of the building.

\subsection{Floor Plan Detection}

\subsubsection{Room Detection}
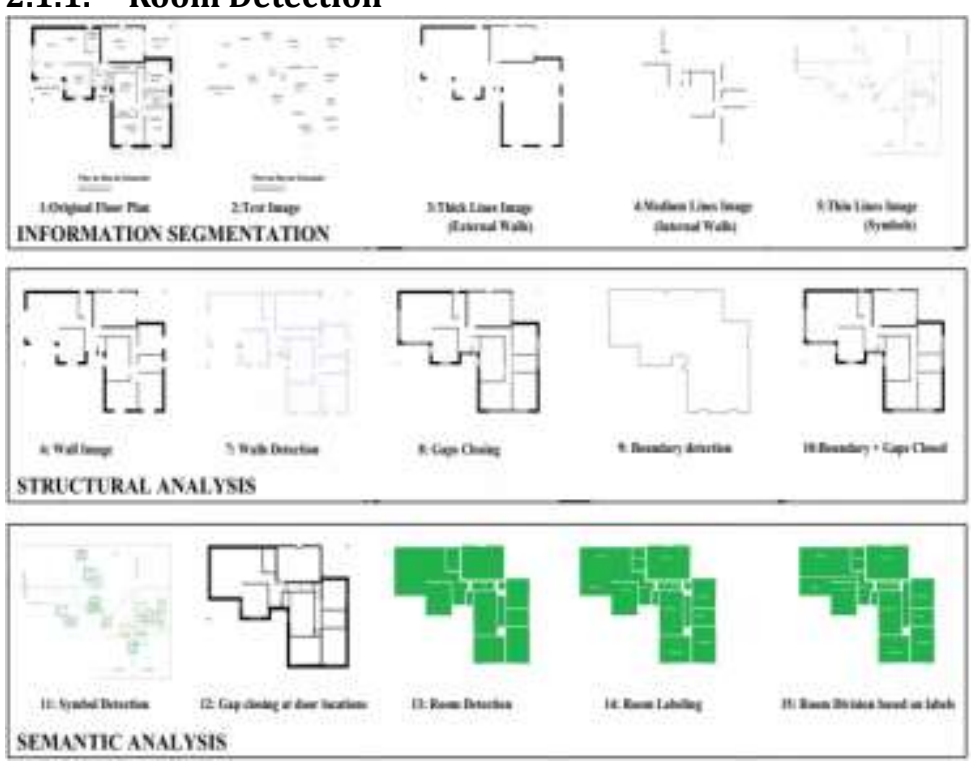

Figure 4. Automatic Room Detection and Room Labeling Workflow

Fig. 4 is a diagram showing the overall flow of the floor plan analysis system. The analysis process begins with a segmentation process that separates various types of information from each other. The structural analysis leading to the work of information segmentation aims to search for the structure of the room. Finally, semantic analysis is applied to improve the structural analysis results and find the sensed spatial function[10].

In order to detect only the shape of the room, a pre-processing process was performed to remove elements that interfere with detection of walls such as desks, chairs, sofas, doors, and watermarks. In addition, unnecessary elements such as text, drawing symbols, and noise from the preprocessed image were removed using morphology conversion.

$$
\begin{aligned}
& \text { dilate }(\mathrm{x}, \mathrm{y})=\max _{(i, j) \in \text { kernel }} \operatorname{src}(x+i, y+i) \\
& \text { erode }(\mathrm{x}, \mathrm{y})=\underset{(i, j) \in \text { kernel }}{\operatorname{minc}(x+i, y+j)}
\end{aligned}
$$

Basically, the morphology transformation uses expansion and erosion operations. Equation (1) is an expansion operation mainly used to recover the reduced size after removing noise, and Equation (2) is an erosion operation mainly used to remove noise. Using the above two operations, the work of removing noise other than the wall in the floor plan was carried out.

\subsubsection{Object Detection}

In the work of detecting the room, the work of finding the wall is carried out, but in the case of doors and stairs, each has its own characteristics. Figure 5 shows examples of training image datasets for Object Detection. In the case of the door, it is usually shaped like the (a) in Fig. 5, and the staircase has the form of a ladder figure as in (b). The detection work was performed using YOLO v2, and the SESYD (Systems Evaluation Synthetic Documents) dataset was used as the image data training to proceed with the detection work[10]. 


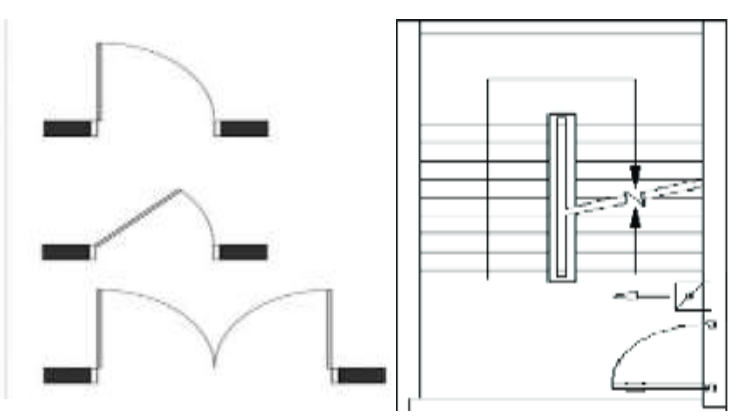

(a)

(b)

Figure 5. Object Detection Training Image : Door(a), Stair(b)

\subsection{Generate Topology Map}

In order to create a topology map, a number of sensor nodes are actually installed on the ceiling of the building. Among the nodes installed in the room, the central node becomes the center node. After that, in order to connect the sensor nodes of the door, hallway, and stairs, the distance between each topology image is firstly determined through the Euclidean distance formula as shown in Equation (3) below.

$$
\mathrm{d}=\sqrt{\left(x_{2}-x_{1}\right)^{2}+\left(y_{2}-y_{1}\right)^{2}}
$$

After calculating the distance of sensor nodes, set the color of each node according to the type of type (room, door, corridor, staircase), and set the sensor node closest to each sensor node as the Closest node as shown in Fig. 6 below. For example, in R1, the closest D2 sensor node among the surrounding door sensor nodes is set as the Closest node.

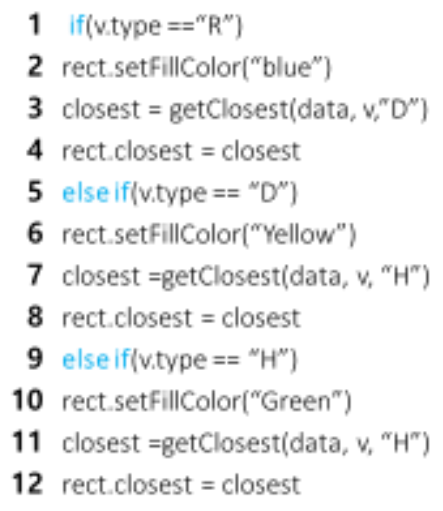

Figure 6. Pseudo code that sets the closest node

\section{3. $\quad$ Result}

Fig. 7 is the result of object detection in the sample floor plan. Except for the wall in Fig. 4, other noises described above were removed, and each door, room, and staircase were detected by learning the dataset in Fig. 5. In addition, data sets such as sofas and desks were also training to detect obstacles. The structure of the room is blue, the structure of the door is yellow, and the structure of the stairs is orange, Obstacles in purple Each Bounding box was created and displayed.

Figure 8 is a picture that shows the sensor node installed in the actual building and located in the center of the room as a Room node, and after setting each sensor node as a Closest node as shown in Fig. 6, it is displayed on the floor plan. After this work is completed, as shown in Fig. 9, the work of connecting the path from the room to the door and from the door to the corridor proceeds. In the sample floor plan below, the structure cannot be moved from room to room, but if there is no corridor node among the sensor nodes closest to the door, it is connected to the other room node closest to the corridor node. Finally, you can move to the map of another floor through the node of the stairs. 


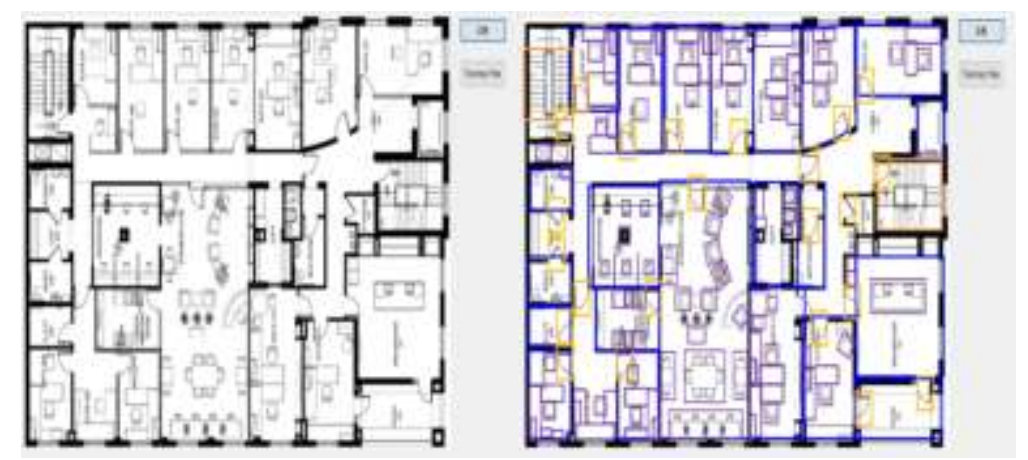

Figure 7. Room, door, and staircase detection results on the floor plan

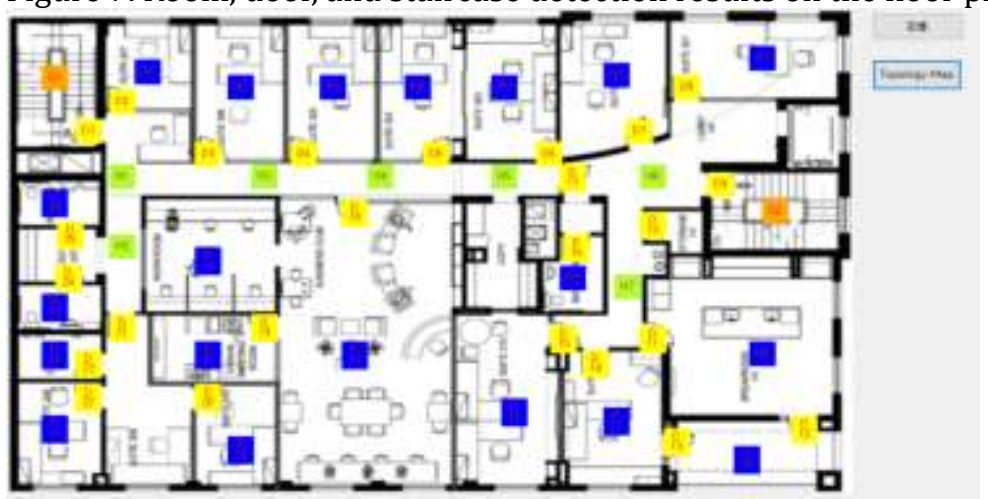

Figure 8. Exhibition of nodes in rooms, doors, Hallway, and stairs

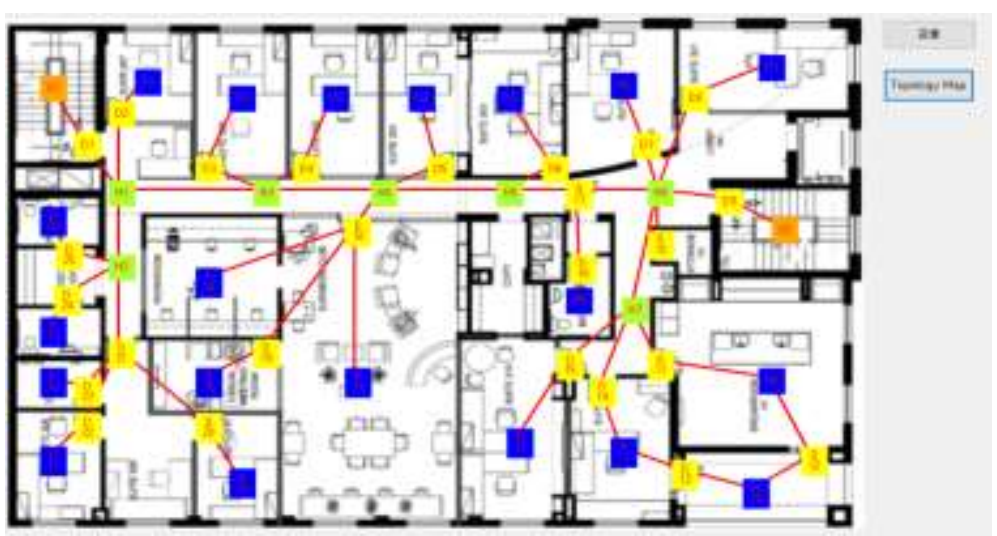

Figure 9. Generate topology map

\section{Conclusions}

In this research, we conduct the process of creating a topology map by overlaying a building floor plan and installed sensors. This method can provide a safe path because the sensors have information if there are no changes of environments or locations of the building. In addition, even though the interior of the building is changed, we can provide that information in the same way as the existing methods through updating change of floor plan. That is, if we have a floor plan image, we can obtain exact structural information regardless of the type of building through the methods of this research.

The biggest advantage of this research is that the route production time is fast. Since the floor plan image of the building is used, there is no need to create an indoor map. SLAM needs a LiDAR sensor detection and comparison to detect rooms and doors. However, our method does not need the LiDAR sensor and we can detect objects quickly since we have the trained images.

When this information is transmitted to the wearable robot, the wearable robot receives information on each path and can walk more stably. In the future, smaller obstacles are also detected while moving and transmitted to the wearable robot so that it can walk avoiding obstacles in the path. In addition, we will conduction additional research to create a path avoiding the wall in the section where the wall and the path overlap. 


\section{Acknowledgements}

This work was supported by the Technology Innovation Program (Robot Industry Core Technology Development Project) (20003914, Development and commercialization of motorized exoskeleton robots for the assistant of daily life exercise) funded By the Ministry of Trade, Industry \& Energy (MOTIE, Korea)

\section{References}

[1] Pons, J. L. Wearable robots: biomechatronic exoskeletons. England : John Wiley \& Sons. 2008

[2] Lee, U. H., Bi, J., Patel, R., Fouhey, D., \& Rouse, E. Image Transformation and CNNs: A Strategy for Encoding Human Locomotor Intent for Autonomous Wearable Robots. IEEE Robotics and Automation Letters, 5(4), 5440-5447, 2020

[3] Jung, G. H., Park, Y. Y., Kim, S. Y., \& Cho, Y. W. The study on how to remove the shadow area for WSN based indoor disaster monitoring system. Cluster Computing, 22(1), 1973-1984, 2019

[4] Tao, Y., \& Ganz, A. Simulation Framework for Evaluation of Indoor Navigation Systems. IEEE Access, 8, 20028-20042, 2020

[5] Li, T., Han, D., Chen, Y., Zhang, R., Zhang, Y., \& Hedgpeth, T. IndoorWaze: A Crowdsourcing-Based Context-Aware Indoor Navigation System. IEEE Transactions on Wireless Communications, 19(8), 5461-5472, 2020

[6] Chen, Y., Leighton, B., Zhu, H., Ke, X., Liu, S., \& Zhao, L. Submap-Based Indoor Navigation System for the Fetch Robot. IEEE Access, 8, 81479-81491, 2020

[7] Jiang, Z., Zhao, J., Han, J., Tang, S., Zhao, J., \& Xi, W. Wi-Fi fingerprint based indoor localization without indoor space measurement. In 2013 IEEE 10th International Conference on Mobile Ad-Hoc and Sensor Systems, 1,384-392, 2013

[8] Tien, M., Park, Y. Y., Jung, K. H., Kim, S. Y., \& Kye, J. E. Performance evaluation on the accuracy of the semantic map of an autonomous robot equipped with P2P communication module. Peer-to-Peer Networking and Applications, 13(2), 704-716, 2020

[9] Ahmed, S., Liwicki, M., Weber, M., \& Dengel, A. Automatic room detection and room labeling from architectural floor plans. In 2012 10th IAPR International Workshop on Document Analysis Systems 339-343, 2012

[10] Delalandre, M., Valveny, E., \& Ramel, J. Recent contributions on the SESYD dataset for performance evaluation of symbol spotting systems. 7423, 198-207, 2011 